\title{
THE PERFORMANCE MANAGEMENT SYSTEM - AN INDIAN PERSPECTIVE
}

\section{DR. KESHAB NANDY}

Professor of Management, NMIMS University, Navi Mumbai, India

\begin{abstract}
Post Globalization, when there was heavy rush of companies from across the globe to set shops in India to take advantage of huge market, there was obviously a wake-up call to the Indian corporates to spruce up and start working "searching for cutting-edge over the competitors". This search for cutting edge led most of them to realize that all other competitive variables being same among the players, the only determinant variable they can bank upon is "Employee Performance" which not being indexed to any "rated capacity" like in a machine, can indeed be harnessed and cultivated to any organizationally designed objective realization process. That being so, Performance Management turned out to be the most researched area of Human Resource Management. But the first goal in this research was how to define performance and then try to measure it since it is a well-recognized fact that what cannot be objectively measured cannot be improved. It was also agreed that once taken to empirical heights, Performance Management System (PMS) should be the only benchmark for administration of any compensation and reward mechanism in any organization. Thus having been choreographed to play the centre-stage role in the survival and growth dynamics of companies, standardizing performance and recording the same on objective lines became the key.

KEYWORDS: Performance Management, Competitive Dynamics, Cutting-Edge, Performance Measurement and Improvement
\end{abstract}

Received: Apr 27, 2019; Accepted: May 17, 2019; Published: Jun 12, 2019; Paper Id.: IJHRMRAUG20191

\section{INTRODUCTION}

Prior to ushering in of competitive dynamics in to the corporate work-culture in the nineties propped by globalization, there was very little sensitivity to competition in the organizations, since in socialistic fervor, being able to provide jobs to a few and developing infrastructure by way of roads, rail, communication etc. were considered to be prime achievements for any industry in India. If after having fulfilled these idealistic socialist goals, some companies could still manage some productivity that was well and good. On the whole, in Indian context, priority was different. Accordingly, not only capacity utilization and machine performance ranked low in priority, there was also least importance attached to employee performance indices. In most organizations, as an annual ritual, confidential-reporting-system was prevalent, wherein the almighty boss will record in a free style manner, good, bad, evil of the subordinates, thus becoming the sole arbiter of the destiny of the subordinates in both career progression and compensation dispensation mechanism. Not for nothing, many exceptionally talented resources were doomed for failure just because for various reasons they could not master the trick of success in this highly subjective process and conversely, many below average performers marched ahead in career growth trampling the highly talented ones. This resulted not only in all round servile culture of nepotism and favoritism getting cultivated in organizations at the cost of productivity, but also the entirely subjective system smacked of bias and prejudice. But with the onset of competitive globalized era, when productivity became the sole criterion for survival and growth, there was a lot of research as to who drives productivity and when it was realized that 
employee performance is the most important driving force, attempts started being made to standardize it on scientific basis in a gradual manner and the process is still on based on feedback and research.

Performance Management is a process of establishing shared understanding about what is to be achieved, and an approach to managing and developing people in a way which increases the probability that it will be achieved in the short and long term (ArmStrong, 1995). PMS is intended to get the pre-designed results from the organizations and the employees working in them with continuing efforts at improving their degrees of performance in measurable terms. Thus, periodic Performance Appraisal of employees is considered one of the most difficult tasks in the manager's job (Feldman,1981). The role of periodic performance appraisal of the employees in an organization has been well recognized and it has been strongly suggested that an effective performance management can yield significant positive benefits to the organizations. (Casico, 1982). While everyone has unambiguously pleaded for a strong role of PMS in the organization's growth process, the opinion of experts largely vary. When promoters/owners opt strongly in favor of PMS fulfilling organizational objectives, the employees largely look at it for fulfilling their career growth expectations, enhancing their morale and ensuring a continuing feel-good factor. Performance Appraisal System in India vary significantly and has evolved greatly from simply no-appraisal to subjective confidential reporting system to 'sophisticated multipurpose, multi component based appraisal system'(Rao, 2004). If we have to look at the effectiveness of PMS in any organization, we need to look at its design, implementation and maintenance phases. Researchers have extensively worked in all these areas in Indian companies but always from a particular stake holder point of view and no comprehensive or generalizable result being available. The present study has been attempted to compile the perceived effectiveness of PMS in an FMCG organization in India. A questionnaire survey was carried out to know the employees' perception of PMS on the basis of critical success factors of PMS which will throw new light on the available knowledge about overall effectiveness of PMS.

\section{LITERATURE REVIEW}

PMS plays the most critical role in the vision and mission realization process of the organization and since this is based on various paradigms of PMS as implemented in the organization, different success factors are drawn to achieve the goals. The review of literature on effectiveness of PMS shows multiple factors of its effectiveness and other aspects as deliberated in the following paragraphs. The validity and reliability of instrument and perceived fairness before and after ratings are important factors of effectiveness of PMS particularly in public sector organizations in India(Mohammed A.H., 2012). However, since performance has turned out to be the sole determinant of success or failure of an organization, be in the public or private sector, it has become imperative that all performance dimensions are clearly defined, performance standardized, properly disseminated and outright transparency maintained to minimize if not fully eliminate subjectivity in performance assessment. But before we talk of several assessment methodologies, we need to keep in mind that performance expectation as derived from the vision statement must have been communicated clearly to all the managers in the hierarchy. Next, these performance parameters will need to be converted to quantifiable goals which is called KRAs(Key Result Area). Unless performance is quantifiable it cannot be measured and "what cannot be measured, cannot be improved". Quantifiable assessment of KRA realization by managers call for periodic review, providing continual feedback and implementing any mid-term course correction, which comprise the core ingredients of a robust performance appraisal system. Using appraisal results for appropriately rewarding performance is another strong pillar of an effective appraisal system. In doing this, the best option always has been to link incentive, rewards or variable salary to the percentage realization of KRAs assigned to a manager. Most of the organizations have gradually moved to a variable salary 
structure which generally comprises 10 to $20 \%$ of the total cost to company of a manager and since this component of salary is directly linked to \% realization of individual KRA and thus that vital component of organizational goal, this not only is the most dynamic matrix of the organizational dynamics but also happens to be the most important factor of realization of vision of an organization. Another important aspect of variable salary mechanism is that it remains mostly differential and very rarely two employees' KRA \% score after the appraisal cycle remains the same and this variable nature of compensation creates a healthy internal competition among the employees thus greatly spurring the organizational productivity as a whole. However, the basic objective of an effective performance appraisal system is exactly not to weed out non-performers always but to identify high performers on the one hand and reward them for reinforcement of high performance and identify low-performers for whom several performance improvement plans including performance counselling, mentoring etc. are planned and implemented since the sole objective of enhancing the effectiveness of performance management system is to improve the employees' performance. (Bob Lavigna, 2010). So addressing the development needs of employees forms an important part of any appraisal system and it is generally approached in a structured manner. One page of the performance appraisal format specifically deals with empowerment initiatives proposed by both reporting officer and reviewing officer of an appraise employee who lists out training, mentoring, counselling and periodic interim assessment needs required to be implemented by HR with a view to converting as many low-performers as possible to the high-performer group. In fact, the efficacy of any performance appraisal system is tested on the strength of having transformed how many low-performers to high-performer category.

It is also a well-known fact that engaged and satisfied employees tend to perform better and are always ready to spend their 'discretionary energy' for the organization resulting in highly effective performance management. Therefore an organization must carry out employee satisfaction survey at periodic intervals preferably through outsiders or student interns so as to obviate the possibility of 'guarded response' if done in-house by HR and consequently the result not being objective. On the basis of periodic employee satisfaction surveys, all efforts should be made to strengthen and reinforce employee satisfaction and engagement to improve effectiveness of performance management system, apart from the efforts like performance improvement plans, suitable on-the-job and off-the-job training, efficient and effective design of appraisal tool, effective communication, feedback and course-correction processes.

Ron Drew S. (2009), found out three main shortcomings of performance management as a narrow framework used to view, analyze and discuss performance ineffective habits of various performers and improperly and inadequately defining the design steps. While suggesting that the effectiveness of PMS can be enhanced by taking care of the above shortcomings, one needs to be careful of being idealistic. So by focusing on critical 20 percent causes, one can solve eighty percent of the performance management related problems which should be the most practical approach. Jim Collins warns that 'People are the most Important asset of your Organization' is indeed misleading and it should be modified as only the right people are the strategic assets of your Organization'(in Crain David, 2009). He rightly emphasized on learning and development for the employees having a right attitude and getting rid of such employees who do not fit in to the bill, a strategy adopted by many including the iconic Wall Mart. So effectiveness of a PMS is decided on two important achievement variables, first, the system by following objective quantifiable methods of performance assessment and measurement through proper KRA identification, not to be confused with generalistic 'job description' and then based on that classify employees to high-performer, low-performer and non-performer categories. Then motivate and encourage high-performers with incentives, rewards and incremental variable salary with high-visibility index, low-performers to be put to learning and development, training, mentoring through a comprehensive performance improvement plan(PIP) with 
periodic intervention and monitoring by tutor officers so that low performers get converted to high performers over a period of time. With regard to such employees who do not show performance improvement in spite of the above PIP as also the category of non-performers as rated on the basis of past two PMS cycles and written communication of deficient attributes calling upon to improve, must be asked to leave as the perseverance policy of the organization. This needs to be done swiftly because any indulgence shown to these non-performers will only drain the organization, a high cost for non-performance by way of loss of productivity, thus affecting the interests of all high performing employees through loss of incentives and profit-sharing.

Segregating the role played by outcomes of effective PMS and factors that can harm it, can be one of the ways to assess the effectiveness of PMS. Hafiz, Zaid and Zaheer (2009), while studying the PMS in public and private sector organizations observed that the factors of effectiveness of PMS include 'émployees knowing how they are performing' and 'Improvement in the accuracy of employees' performance' and the factors detrimental to PMS include 'Organizational politics affecting the performance of targeted employees', 'Doubts in the minds of employees about the after effects of appraisal', 'Appraisal's focus on short term goals' and subjectivity of appraisal process.

The factors of effectiveness of PMS can be categorized in to personal factors and organizational factors (Wood and Marshal, 2008). The personal factors included 'The appraiser experience' and 'Training' while the organizational factors included management concern, accountability, instrument adequacy, instrument sensitivity and importance of appraisal.

Chandrasekhar S.F., 2007 while studying the perceived effectiveness of performance appraisal system (PAS) in an engineering company had found that perception of employees about effectiveness of PMS varies though not very significantly, with their job levels and personal factors. The higher level of employees (managers) are more likely to respond positively about effectiveness of PMS of the organization than their counter parts at lower levels and possible reasons for this variation may be their depth of understanding of PMS and its consequences due to their higher qualifications and organizational exposure.

Despite the universal realization that performance is the key to organizational success and continuing extensive research in the field, the questions that have yet to be more rigorously pursued in the literature include: what leads to Performance Appraisal System (PAS) effectiveness and how can PAS be defined? How can organizations understand if their pursued PAS is effectively producing their desired results? Research shows that the measures and systems we now have, are not free from bias (Cleveland \& Murphy, 1992; Dewberry, 2001;Scott \& Einstein, 2001; and Lam \& Schaubroeck,1999 in Anderson, 2002). The biggest challenge in present times has come after vertical linkage of KRA score ratings in PAR to an employee's compensation package, be it variable salary or other forms of financial incentives. It has been observed that when there is incremental percentage growth in number of high performers as assessed by PAS, it does not commensurately result in the increase in the productivity of the organization and in a few cases results in negative growth. This has resulted in organizations' pessimism that the PAS is not an entirely objective process. Thus many organizations have resorted to Bell-Curve method as basis for compensation and benefits for employees which has naturally dampened the efficacy of performance management system. However, when more study and research is called for to make PAS much more objective, there is no denying the fact that performance management system is a dynamic behavioral tool. With organizations continually facing new competition and challenges, PMS should be reviewed and updated to meet the emerging requirements, introduce intervention programmes or new changes in strategic framework 
from time to time. Similarly, the success factors for every organization is different making it imperative that organizations keep customizing PMS keyed to its changing strategy. It should provide specific measures to achieve its vision and objectives within a specified timeframe. The attempt should be made to identify as many common factors of effectiveness of PMS as possible so that the bottlenecks in customization can be significantly reduced.

\section{OBJECTIVES}

To identify the perceptual process of various levels of employees in an organization of the efficacy of PMS for career growth and incremental compensation package.

\section{METHODOLOGY}

The present study was conducted in a leading Indian liquor company named as Tilaknagar Industries Ltd. A questionnaire survey was conducted using structured questions having items ranging from organizational factors to factors relating to characteristics, design, process and linkages of appraisal results to career growth and compensation and benefits of the employees.

\section{Data Collection Design}

The study is based on primary data. However, secondary data about the organization were also used as parameters. The primary data was collected in terms of responses to the questions asked in the questionnaire survey while secondary data were collected from the company related literature and website.

\section{Instrument}

A structured questionnaire was used for survey comprising a list of items pre-validated through experts' opinion (factors of perception recorded level-wise about effectiveness of PMS) divided in to seven categories like Organizational factors, Design and features of PMS, Linkage of PMS features and KRAs with organizational productivity, Factors of processes of PMS, Perception index of employees of different levels about PMS as tool, Use of Appraisal results for career growth and Use of appraisal results for grant of compensation and benefits. The questionnaire was pretested on ten subjects across different levels to check and be sure of its reliability.

\section{Sample Design}

Looking in to the constraints of paucity of time with respondents, convenience quota sampling was used to gather the response from the subjects. Ten percent of total employee strength was taken as a sample. This sample was drawn from different categories of employees in proportion to their strength in a particular category. The sample size was 29 . The profile of the respondents is as depicted in the following table.

Table 1

\begin{tabular}{|c|c|c|c|c|c|c|c|c|c|c|c|}
\hline \multicolumn{3}{|c|}{ Qualification } & \multicolumn{3}{|c|}{ Age (Yrs) } & \multicolumn{3}{|c|}{ Designation } & \multicolumn{3}{|c|}{ Experience ( No. of Yrs) } \\
\hline Category & No & $\%$ & Category & No & $\%$ & Category & No & $\%$ & Category & No & $\%$ \\
\hline Non-Graduate & 7 & 24.13 & $20-30$ & 6 & 20.68 & Exe+Sales Exe & 4 & 13.79 & $0-5$ yrs & 7 & 24.13 \\
\hline Degree & 7 & 24.13 & $31-40$ & 12 & 41.37 & AsstMgr & 9 & 31.09 & $6-10 \mathrm{yrs}$ & 10 & 34.48 \\
\hline $\mathrm{PG}$ & 4 & 13.79 & $41-50$ & 6 & 20.68 & DyMgr+Mgr & 5 & 17.24 & $11-15 \mathrm{yrs}$ & 5 & 17.24 \\
\hline $\mathrm{MBA} / \mathrm{CA}$ & 2 & 6.89 & Above 50 & 5 & 17.24 & DGM/GM/VP & 9 & 31.09 & $16-20$ yrs & 3 & 10.34 \\
\hline $\mathrm{MBA} / \mathrm{CA} / \mathrm{PhD}$ & 9 & 31.09 & & & & Top Mgt & 2 & 6.89 & $21-25$ yrs & 3 & 10.34 \\
\hline & & & & & & & & & Above 25 yrs & 1 & 2.94 \\
\hline
\end{tabular}


Nearly one fourth $(24.13 \%)$ of the respondents are Matriculates but non-Graduates which is the lowest level of employee cadre in the organization. They are in the age group of 20-30 years and possess work experience ranging from 0-5 years. There appears to be some bias in response from this category of employees owing to two possible reasons: one, their educational qualification and two their lack of perceived linkage between tangible benefits and PMS. An equal percentage of employees are in the age group of 31-40 years and are degree holders which is considered to be lowest level blue collared employees and their experience range is 6-10 years. They are either bottom ranked supervisors or executives themselves. This category of employees has a reasonably good understanding of PMS and therefore chances of any biased response from them are less compared to the non-graduate base level employees. About twenty percent (20.68\%) are post graduate degree holders having experience of 11-15 years and are at the level of deputy managers or managers. They are expected to have very good understanding of PMS and its linkage to organizational compensation and benefits policy. Nearly one third $(31.09 \%)$ of the respondents are MBA/CA/Ph.Ds having experience ranging from 21-25 years which presupposes their clear understanding of the organization's PMS policy and how it has a relationship and linkage with the organization's compensation policy. Hence response from this category is likely to be with very minimal error.

\section{RESULTS AND DISCUSSIONS}

The result was clear and unanimous that the efficacy of PMS is best validated by improved bottom-line of any organization. Communication plays a critical role in familiarizing all levels of employees especially at lower levels about the benefits of PMS and how it plays key role in organizational growth matrix, keeping employees in a continual state of high motivational level, greater job satisfaction, heightened sense of belonging, developing a culture of innovation and creativity and most particularly by bringing in a culture of healthy internal competition by linking PMS rating and KRA score to a differential compensation mechanism in the organization. Intensive training to the appraisee and appraiser on identification of KRAs is very essential because it has been observed that both generally confuse job description with KRA whereas KRA forms only the quantifiable result part of the job description. Then taking in to account the key performance indicators of an employee as per his job profile in the organization, how to rank weightages to the 4-5 KRAs identified for an employee, needs good communication and on the job training for all. How to do interim assessment of KRA realization within an appraisal cycle leading to mid-course correction through performance improvement programmes, counseling etc. are other grey areas to be well covered.

\section{CONCLUSIONS}

Contemporary PMS models as practised in different organizations have indeed come a long way from the earlier days of purely subjective and grossly biased confidential reporting system. Several attributes like self-appraisal, 360 degree, KRA based assessment, balanced score card, assessment centre mechanism etc. have definitely ascribed a lot of transparency and objectivity to the system. Nevertheless, since PMS deals with human beings, subjectivity cannot be eliminated altogether though it has been reduced remarkably. Inevitable challenges; and therefore great opportunities surely call for further research and validation. 


\section{REFERENCES}

1. Agbola et al (2011), “The Effectiveness of Performance Appraisal as a Tool for Enhancing Employee Performance in the Public Health sector”, Global Management Journal

2. Amy Tung, Kevin Baird, Herbert P. Schoch, (2011) "Factors influencing the effectiveness of performance measurement systems", International Journal of Operations \& Production Management, Vol. 31 Issue: 12, pp.1287 - 1310

3. Armstrong M.A.(2000), 'A Handbook of Human Resource Management Practice”, Kopan Page, London, pp 411-12

4. Procházka, D. A. V. I. D., \& Ilinitchi, C. R. I. S. T. I. N. A. (2009). Adoption of IFRS and its impact on the financial and management accounting: a case from the Czech Republic. In Audit and Accounting Convergence Conference.

5. Biron,Farndale and Paauwe(2011), "Performance Management effectiveness: Lessons from world's leading firms", The International Journal of Human Resource Management, Vol 22, No 6,1294-1311

6. Bob Lavigna(2010)," Driving Performance by Building Employee Satisfaction and Engagement”, (US)Government finance Review Feb 2010

7. Bonadio(2009)," HR Field Guide: 5 Tips For Getting The Most Out Of Your Workforce Performance Management System”( http://ssrn.com/abstract=1408762)

8. Chandra ShekharS.F.(2007),"Assessment of Effectiveness of Performance Appraisal System: Scale Development and its Usage”,ISSN 133, Vol !, Issue 3/4

9. Crain David W.( 2009)," Only right people are strategic Assets of Organizations", STRATEGY AND LEADERSHIP, Vol 37, No 6

10. Feldman, J. M. (1981)," Beyond attribution theory: Cognitive processes in performance evaluation”. Journal of Applied Psychology, 66, 127-148.

11. Fletcher C.(2001)," Performance Appraisal and Management :the developing research agenda, Journal of Occupational and Organizational Psychology, Vol 74, pp 474-483.

12. Gutierrez, M. M. (2018). Characteristics, Managerial Skills and Job Performance of Women Middle Managers in Central Luzon (Region III), Philippines. International Journal of Human Resources Management (IJHRM) ISSN (P), 2319-4936.

13. Hafiz MuhammadIshaq, Muhammad Zahid Iqbal and ArshadZaheer (2009), "Effectiveness of Performance Appraisal: Its Outcomes and Detriments in Pakistani Organizations”, European Journal of social sciences, Vol 10, November 2009

14. Jonathan R. Anderson(2002)," Measuring Human Capital: Performance Appraisal Effectiveness", Midwest Academy of Management Conference

15. Joyce Tand Stivers B (2000)," Building a Balanced Performance Management”, Advanced Management Journal. 65 No 2

16. Krishnan, D. S. G., \& Rajesh, A. (2018). Gulf Cooperation Council Banking Performance Analysis-An Exploratory Study. IMPACT: International Journal of Research in Humanities, Arts and Literature (IMPACT: IJRHAL) ISSN (P), $2347-4564$.

17. Mohammed A.H.,(2012), "Performance Appraisal System of Bangladeshi Civil Service: An Analysis of its Efficacy”, IPMR

18. Murphy K.R. and Cleaveland, J.N.,(1995),"Understanding Performance Appraisal: Social, Organizational and Goal Based Perspectives, Sage, Newbury Park CA 
19. Ouada Jamoussi, H. B., \& Makhlouf, E. M. (2018). School Performance and the Education Systems' Efficiency: An International Comparison Based on OECD PISA Surveys. International Journal of Human Resources Management (IJHRM) $\operatorname{ISSN}(P), 2319-4936$.

20. Rao T.V.(2004),"Performance Management and Appraisal Systems:HR Tools for Global Competitiveness", Response books, New Delhi,pp 213-15

21. Ron Drew Stone(2009),"Achieving Performance Results Through Performance Centred Design framework”, Performance Improvement, Vol 48 No 5

22. Dr. Keshab Nandy(2017), "Be a Habitual Winner", Himalaya Publishing House, Mumbai. 